\title{
A New Multi-Body Dynamic Model of a Deep Ocean Mining Vehicle-Pipeline-Ship System and Simulation of Its Integrated Motion
}

\author{
Yu Dai 1,2,3,* - Liping Pang 1 - Lisong Chen ${ }^{1}$ - Xiang Zhu1 - Tao Zhang ${ }^{1}$ \\ ${ }^{1}$ Central South University, College of Mechanical and Electrical Engineering, China \\ 2 Shanghai Jiao Tong University, State Key Laboratory of Ocean Engineering, China \\ ${ }^{3}$ Central South University, Shenzhen Research Institute, China
}

In this paper, a three-dimensional multi-body dynamic model of a seafloor mining vehicle is developed in the RecurDyn simulation program by integrating the developed terramechanics model of the seafloor sediment. The discrete element method (DEM) is employed to model a mining pipeline as discrete rigid elements linked by flexible connectors. An innovative user-defined subroutine for parametrically and rapidly automatically building a DEM model for such long pipeline is developed in the C\# language in RecurDyn. A new 3D multi-body dynamic model of a total deep ocean mining vehicle-pipeline-ship system is established with the integration of developed models for subsystems. The integrated motion simulations are achieved and demonstrate that the synchronized stable motion of the total system can be well maintained during operation processes.

Keywords: deep ocean mining system, terramechanics model, multi-body dynamic model, discrete element model, user-defined subroutine, integrated motion simulation

Highlights

- A multi-body dynamic model of a seafloor mining vehicle has been developed by integrating an innovative user-defined subroutine for characterizing the terramechanics model of the seafloor sediment.

- An innovative user-defined subroutine for parametrically and automatically building a multi-body discrete element model of an extremely long mining pipeline has been developed.

- A new multi-body dynamic model of a deep ocean mining vehicle-pipeline-ship system has been established.

\section{O INTRODUCTION}

Given the gradual reduction of terrestrial resources, the rich mineral resources of the deep seafloor have excited great interest and are likely to be commercially exploited in the near future. An integrated system with a seafloor-tracked mining vehicle, flexible hoses, a buffer, lifting pipes and a surface mining vessel is considered to be the most promising continuous mining system [1], as shown in Fig. 1.

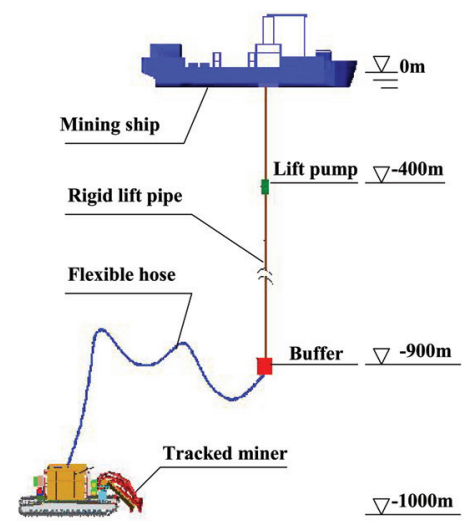

Fig. 1. Schematic diagram of a $1000 \mathrm{~m}$ deep ocean mining system
To analyse the mobility of tracked mining vehicles on the seafloor, Dai et al. [2] and [3] built a seafloortracked mining vehicle as a single-body vehicle model with mesh elements of track-sediment interactions, as well as a multi-body dynamic model, and performed various operational simulations. Additionally, research has been conducted for modelling tracked vehicles on land surface soils [4] and [5]. Rubinstein and Coppock [5] used the program LMS-DADS to develop a multi-body model of tracked vehicles and applied user-defined forces to represent the trackterrain interactions. Gao et al. [6] and Wong et al. [7] and [8] developed two simulation programs, RTVPM and NTVPM, for parametrically modelling, analysing and evaluating tracked vehicles with long-pitch tracks and flexible tracks.

To evaluate the dynamics of the pipeline as well as the total ocean mining system, Hong et al. [9] developed a method for 3D nonlinear dynamic analysis of marine pipes. Rustad et al. [10] used the finite element method to build a mathematical model of an ocean pipeline system. Brink and Chung [11] carried out a computer simulation for the dynamic positioning control of a large ocean mining ship-pipe 
system. Hong and Kim [12] performed analysis on the coupled dynamics of a tracked vehicle and flexible riser.

Thus far, however, the complex dynamic characteristics of the total deep ocean mining system have not yet been ascertained; therefore, its design scheme and continuous operation performance have yet to be evaluated, as it is costly and extremely difficult to perform in-situ ocean tests. The dynamic simulation is an effective solution. Accurate and efficient dynamic models for subsystems and then integrated into the total system have been developed in this paper.

\section{TERRAMECHANICS MODEL OF THE SEAFLOOR SEDIMENT}

As seafloor in-situ terramechanics tests are extremely difficult to perform, laboratory-simulated experiments were conducted. Fig. 2 shows the total experimental system.

A series of pressure-sinkage and shear experiments was performed, and corresponding experimental curves were obtained [3], as shown in Fig. 3.

The pressure-sinkage experimental curves can be characterized by Bekker's exponential equation, as follows [13]:

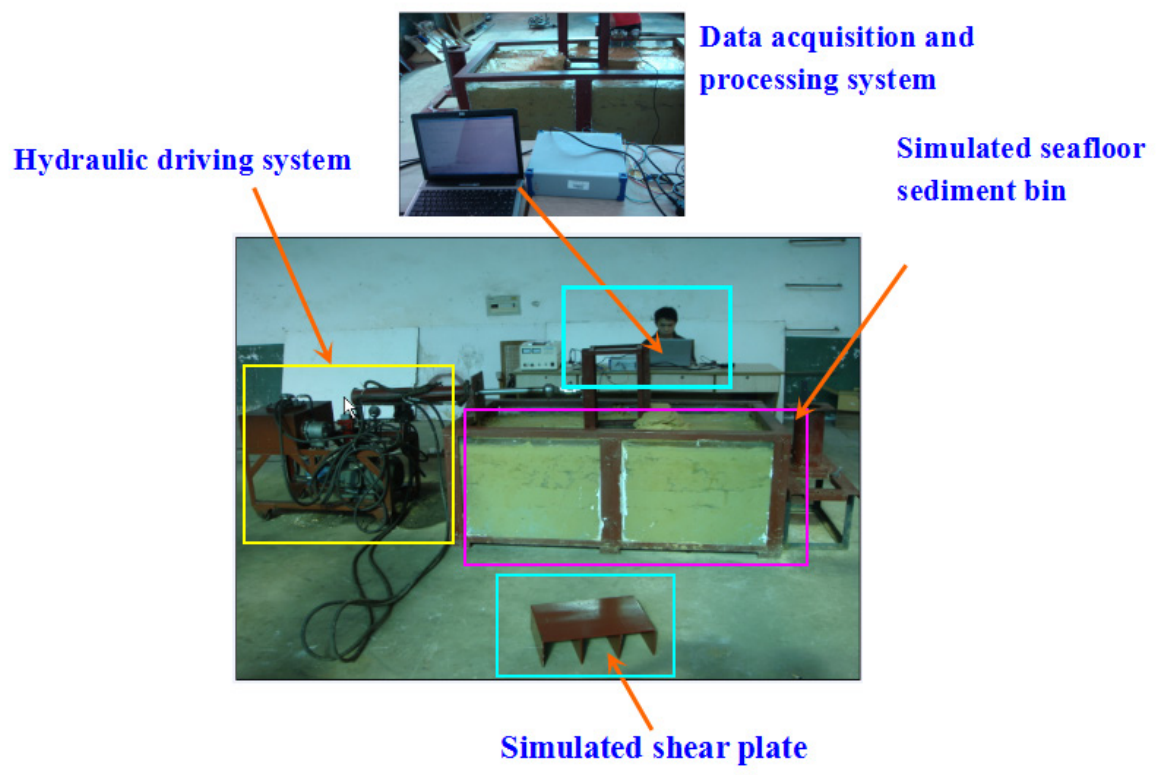

Fig. 2. Photo and illustration of the terramechanics experimental system
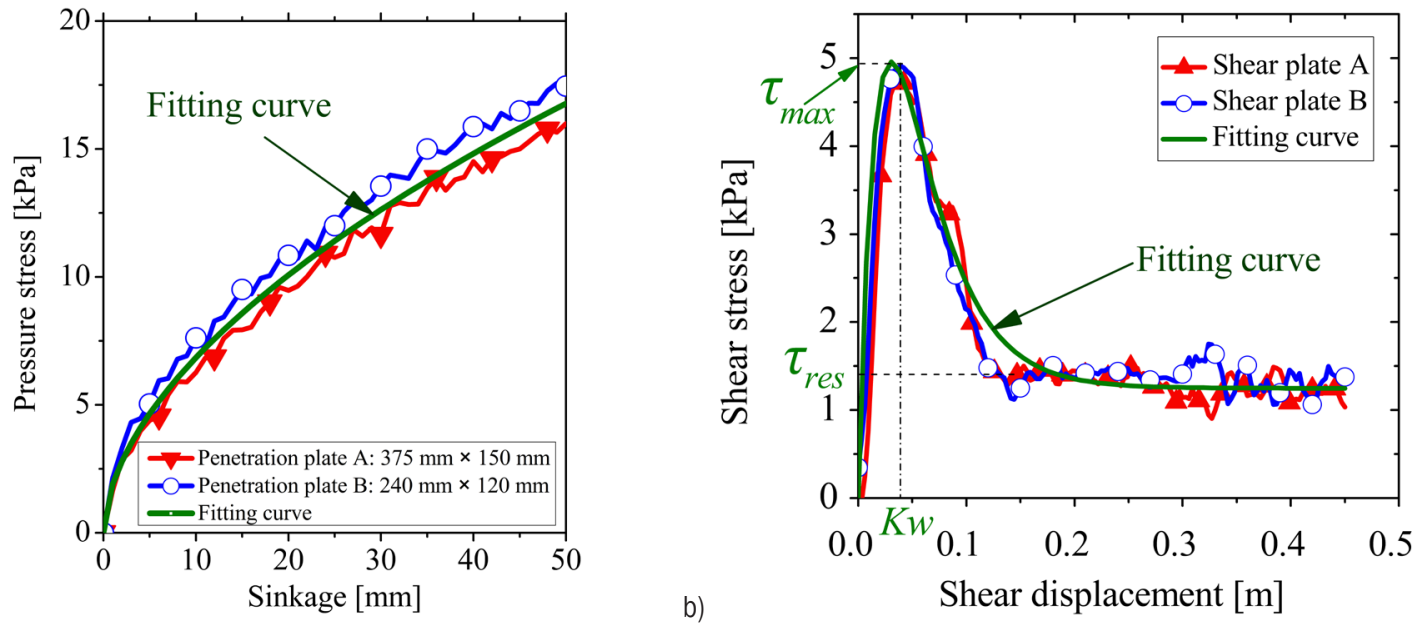

Fig. 3. Experimental curves and fitting curves: a) pressure-sinkage; b) shear stress- displacement 


$$
p=\left(\frac{k_{c}}{b}+k_{\phi}\right) z^{n}
$$

where $p$ is the normal pressure; $b$ is the width of the track; $k_{c}$ and $k_{\phi}$, are the soil cohesive and friction modulus, respectively; $z$ is the sinkage; and $n$ is the soil deformation exponent.

The values $k_{c}, k_{\phi}$ and $n$ in the Eq. (1) can be derived from the results of a minimum of two tests with two sizes of penetration plates, as illustrated in Fig. 3a. The tests produced two curves:

$$
\left\{\begin{array}{l}
p_{1}=\left(k_{c} / \mathrm{b}_{1}+k_{\phi}\right) z^{n} \\
p_{2}=\left(k_{c} / \mathrm{b}_{2}+k_{\phi}\right) z^{n}
\end{array} .\right.
$$

On the logarithmic scale, Eq. (2) can be rewritten as:

$$
\left\{\begin{array}{l}
\log p_{1}=\log \left(k_{c} / \mathrm{b}_{1}+k_{\phi}\right)+n \log z \\
\log p_{2}=\log \left(k_{c} / \mathrm{b}_{2}+k_{\phi}\right)+n \log z
\end{array} .\right.
$$

They represent two parallel straight lines with nearly same slopes on the log-log scale. The exponent of deformation $n$ can be determined from the slopes of the straight lines. At the sinkage $z=1$, the value of the normal pressure for the two sizes of plates are:

$$
\left\{\begin{array}{l}
\left(p_{1}\right)_{z=1}=k_{c} / \mathrm{b}_{1}+k_{\phi}=K_{1} \\
\left(p_{2}\right)_{z=1}=k_{c} / \mathrm{b}_{2}+k_{\phi}=K_{2}
\end{array} .\right.
$$

In Eq. (4), $b_{1}$ and $b_{2}$ are known values as the widths of the penetration plates; $K_{1}$ and $K_{2}$ are measured values; and the only unknowns are $k_{c}$ and $k_{\phi}$. Thus, two different sizes of plates were used in the pressure-sinkage experiments, numbered as penetration plates $A$ and $B$, as illustrated in Fig. 3a.

On the log-log scale, the curves in Fig. 3a were expressed in Fig. 4:

The slopes of line $A$ and line $B$ in Fig. 4 were ca. 0.54 and 0.58 . As mentioned above, the exponent of deformation $n$ can be determined from the average value of the slopes of these two lines as $n=0.56$.

It was also determined from Fig. 4 that when the sinkage $z=1 \mathrm{~mm}$, the coordinate values of intersection points of line $A$ and line $B$ with the pressure axis were about 2.11 and 1.65 , respectively. Converting the unit of the sinkage from millimetre to metre, while considering the exponents of deformation were 0.54 and 0.58 , the following equations can be derived:

$$
\left\{\begin{array}{l}
k_{c} / \mathrm{b}_{1}+k_{\phi}=87.96\left(\mathrm{kPa} / \mathrm{m}^{\mathrm{n}+2}\right) \\
k_{c} / \mathrm{b}_{2}+k_{\phi}=90.67\left(\mathrm{kPa} / \mathrm{m}^{\mathrm{n}+2}\right)
\end{array} .\right.
$$

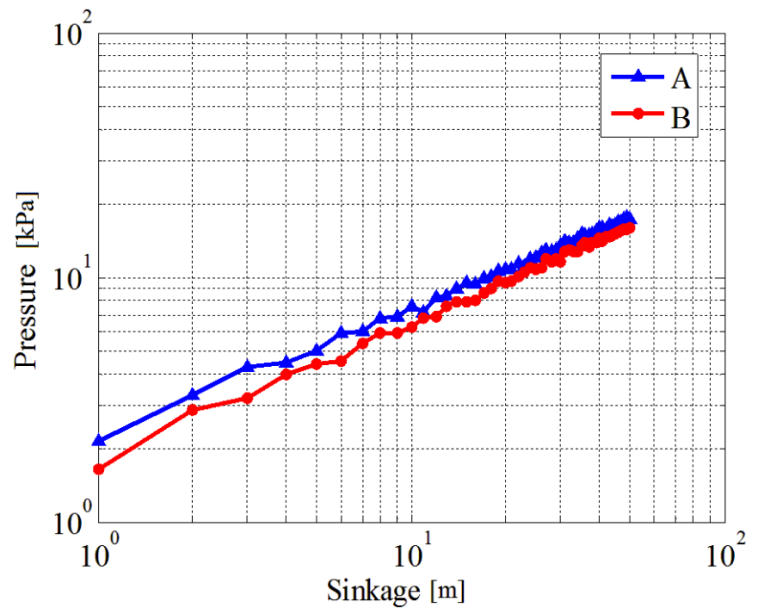

Fig. 4. Pressure stress-sinkage experiments curves on the logarithmic coordinate

Substituting the known values $b_{1}=0.15, b_{2}=$ 0.12 into Eq. (5), then $k_{c}=1.62 \mathrm{kN} / \mathrm{m}^{\mathrm{n}+1}, k_{\phi}=77.1$ $\mathrm{kN} / \mathrm{m}^{\mathrm{n}+2}$ can be obtained.

The observed shear property is different from most of the land surface soils [14]. However, Wong's equation can be fitted well [15]:

$$
\tau=\tau_{\max } K_{r}\left\{1+\left[\frac{1}{K_{r}\left(1-e^{-1}\right)}-1\right] e^{1-j / K_{\omega}}\right\}\left(1-e^{-j / K_{\omega}}\right),
$$

where $\tau_{\max }$ is the maximum shear stress, $K_{r}$ is the ratio of the residual shear stress $\tau_{\text {res }}$ to $\tau_{\max }$, and $K_{\omega}$ is the shear displacement when $\tau_{\max }$ occurs. Observed from the shear experimental curves in Fig. $3 b$, the above parameters can be obtained directly.

\section{MULTI-BODY DYNAMIC MODEL OF A SEAFLOOR MINING VEHICLE}

A multi-body dynamic simulation model of a seafloortracked mining vehicle has been established with the RecurDyn simulation program [3], as shown in Fig. 5.

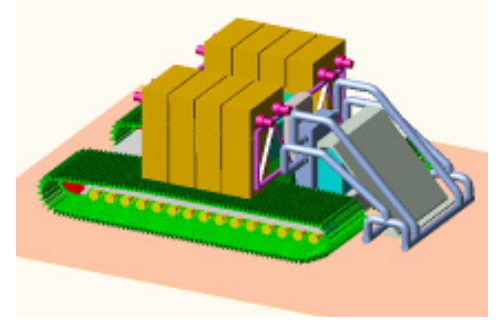

Fig. 5. 3D multi-body dynamic simulation model of a seafloortracked mining vehicle

A user-defined subroutine for characterizing the specific terramechanics models of the seafloor 
sediment was developed using the $\mathrm{C}$ language in the Visual Studio.Net environment and then integrated into the RecurDyn/Track simulation environment.

\section{MULTI-BODY DEM MODEL OF A MINING PIPELINE}

The pilot ocean mining pipeline is composed of a 900 $\mathrm{m}$ long rigid lifting pipe and a $400 \mathrm{~m}$ long flexible riser. Its nonlinear dynamic characteristics, such as large displacement and small deformation, should be taken into account. There were three main modelling methods for dynamic analysis of a pipeline: finite element method (FEM), lumped parameter method (LPM) and discrete element method (DEM). For the FEM, its computation is relatively large and computational time is extremely long. The LPM has a clear shortcoming in simulating the anti-bending and anti-torque performances of a pipeline. However, the DEM can describe well the large displacement and large rotation of a pipeline, and it can significantly reduce the computation as its stiffness matrix is a highly sparse matrix.

The long mining pipeline was discretized into a certain number of rigid elements linked by flexible connectors. The flexible connector was expressed by six spring elements. The external hydrodynamic loads were applied to the corresponding discrete rigid elements.

The stiffness coefficients for the flexible connector were the axial spring stiffness $k_{x}$, lateral spring stiffness $k_{y}$ and $k_{z}$, torsional spring stiffness $k_{r x}$, and rotational spring stiffness $k_{r y}$ and $k_{r z}$, which can be expressed as:

$$
\left\{\begin{array}{l}
k_{x}=E A / L ; k_{y}=12 E I_{z} / L^{3} ; k_{z}=12 E I_{y} / L^{3} \\
k_{r x}=G I_{p} / L ; k_{r y}=E I_{y} / L ; k_{r z}=E I_{z} / L
\end{array},\right.
$$

where $E$ and $G$ are Young modulus and shear modulus; $A$ and $L$ are cross-section area and length; $I_{y}$ and $I_{z}$ are second moments of area; $I_{p}$ is the polar moment of inertia.

The external hydrodynamic force can be calculated as:

$$
F_{h}=-\frac{1}{2} C_{d} \rho_{w} d\left|v_{c}-v_{p}\right|\left(v_{c}-v_{p}\right)-\frac{\pi d^{2}}{4} C_{m} \rho_{w}\left(\dot{v}_{c}-\dot{v}_{p}\right),
$$

where $d$ is the outer diameter of pipe, $v_{p}$ and $\dot{v}_{p}$ are pipe's velocity and acceleration, respectively.

The RecurDyn commercial program was used to model the long pipeline. The bushing element available in RecurDyn can be used to model the flexible connector between rigid elements. The bushing element defines a three-direction force and a three-direction moment to retrain and connect rigid elements, and its mechanical model can be expressed as:

$$
\begin{aligned}
& \left(\begin{array}{l}
F_{x} \\
F_{y} \\
F_{z} \\
T_{x} \\
T_{y} \\
T_{z}
\end{array}\right)=\left(\begin{array}{cccccc}
K_{11} & 0 & 0 & 0 & 0 & 0 \\
0 & K_{22} & 0 & 0 & 0 & 0 \\
0 & 0 & K_{33} & 0 & 0 & 0 \\
0 & 0 & 0 & K_{44} & 0 & 0 \\
0 & 0 & 0 & 0 & K_{55} & 0 \\
0 & 0 & 0 & 0 & 0 & K_{66}
\end{array}\right)\left(\begin{array}{c}
x \\
y \\
z \\
\theta_{1} \\
\theta_{2} \\
\theta_{3}
\end{array}\right)+ \\
& +\left(\begin{array}{cccccc}
C_{11} & 0 & 0 & 0 & 0 & 0 \\
0 & C_{22} & 0 & 0 & 0 & 0 \\
0 & 0 & C_{33} & 0 & 0 & 0 \\
0 & 0 & 0 & C_{44} & 0 & 0 \\
0 & 0 & 0 & 0 & C_{55} & 0 \\
0 & 0 & 0 & 0 & 0 & C_{66}
\end{array}\right)\left(\begin{array}{l}
V_{x} \\
V_{y} \\
V_{z} \\
\omega_{1} \\
\omega_{2} \\
\omega_{3}
\end{array}\right)-\left(\begin{array}{l}
F_{1} \\
F_{2} \\
F_{3} \\
T_{1} \\
T_{2} \\
T_{3}
\end{array}\right),
\end{aligned}
$$

where $K_{i i}$ and $C_{i i}(I=1 \sim 6)$ are stiffness and damper coefficients, $x, y, z$ represent the translational displacements between adjacent rigid elements; $\theta_{1}$, $\theta_{2}, \theta_{3}$ represent the rotational displacements between adjacent rigid elements; $V_{x}, V_{y}, V_{z}$ are relative velocities; $\omega_{1}, \omega_{2}, \omega_{3}$ are rotational velocities; $F_{1}, F_{2}$, $F_{3}, T_{1}, T_{2}, T_{3}$ are preloads. The stiffness coefficients in the bushing element can be determined by the equivalent spring coefficients in Eq. (7) for the multibody discrete element model.

As the DEM model for such long pipeline has a large number of discrete rigid elements associated with discrete hydrodynamic forces and flexible connectors, it is extremely time-consuming and likely to cause mistakes to build the DEM model one-by-one for each discrete element by the traditional method. Therefore, an innovative user-defined subroutine for parametrically and rapidly automatically building of the DEM model was developed in the $\mathrm{C \#}$ language in RecurDyn, based on its secondary development platform ProcessNet.

Three sub-functions were created respectively for the discrete rigid elements, flexible connectors, and discrete hydrodynamic forces. After compiling the $\mathrm{CA}$ program in the ProcessNet and implementing the three sub-functions in RecurDyn, the corresponding multi-body DEM model of the mining pipeline can be established efficiently and accurately. 


\section{KINEMATICS MODEL OF A MINING SHIP}

In this paper, only the heave motion characteristic of the mining ship induced by the surface wave was considered; however, its dynamics characteristics caused by the pipeline were neglected. The heave motion characteristic of the mining ship can be described as:

$$
z_{h}=z_{h a} \sin \left(\frac{2 \pi}{T} t\right)
$$

where $z_{h}$ and $z_{h a}$ represent the ship's heave displacement and heave amplitude, respectively; $z_{h a}$ is determined by the ship structure, wave height, etc.

According to the Moor empirical equation, the heave amplitude $z_{h a}$ can be approximately computed as:

$$
\begin{aligned}
2 z_{h a}= & A_{0}+A_{1} C_{W}+A_{2} C_{B}+A_{3} \frac{L}{B}+A_{4} \frac{d}{L}+ \\
& +A_{5} L_{C B}+A_{6} \frac{K_{Y Y}}{L}+A_{7} \frac{V}{\sqrt{L}},
\end{aligned}
$$

where $C_{W}$ is the coefficient of the water surface profile; $C_{B}$ is the square coefficient; $L$ is the ship length; $B$ is the ship width; $d$ is the ship draft; $L_{C B}$ represents the longitudinal position of the centre of the buoyancy; $V$ is the speed of the ship; $K_{Y Y}$ is the longitudinal mass inertia radius; $A_{0}, A_{1}, \ldots, A_{7}$ are coefficients determined by the wind scale and ship length.

\section{SIMULATIONS OF A TOTAL MINING SYSTEM}

Integrating the above-developed models for subsystems in the RecurDyn environment, a new 3D multi-body dynamic simulation model of the total deep ocean mining vehicle-pipeline-ship system was formed.

In the actual operation, it was designed so that the mining vehicle moved along the predetermined paths on the seafloor; simultaneously, the ship towed the total pipeline subsystem to follow the vehicle, which was referenced for the dynamic simulation of the operation process of the total system in the paper.

Longitudinal straight-line motion is the most typical and common integrated motion state of the total system. For this simulation, the input velocity for the mining vehicle was set to $0.6 \mathrm{~m} / \mathrm{s}$, while the mining ship was subjected to a the same towing velocity along the longitudinal direction. Fig. 6 shows the displacements of subsystems.

The motion states of all the subsystems were stable and the synchronized motion of the total system can be well maintained. The actual velocity of the vehicle was a little less than its input velocity as a slip on the seafloor was inevitable.

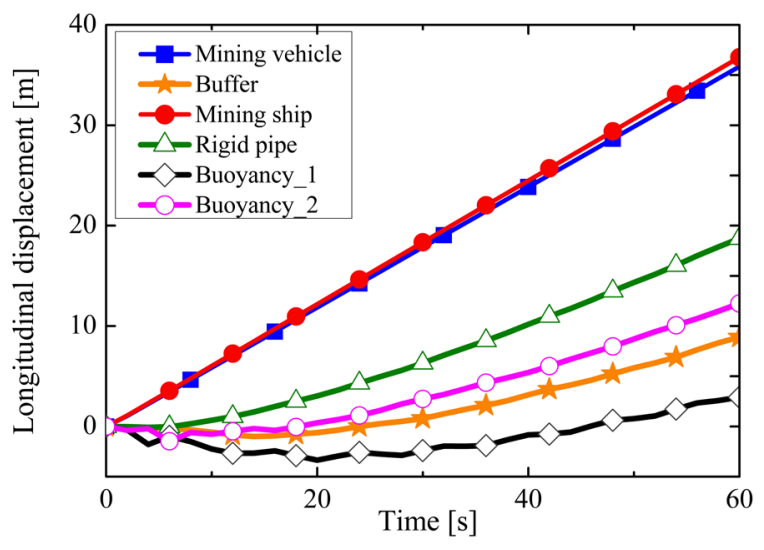

Fig. 6. Displacements simulations of subsystems for the longitudinal straight-line motion
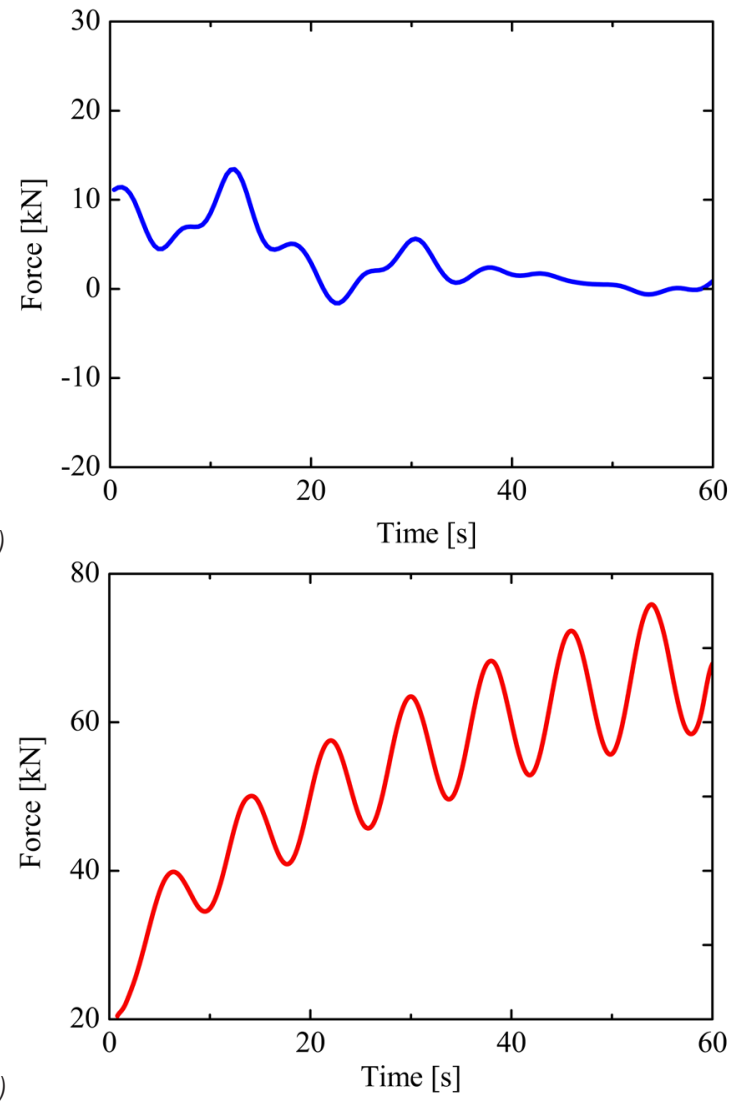

Fig. 7. Interaction forces between subsystems: a) Iongitudinal force on the vehicle by the pipeline; b) longitudinal force on the pipeline by the ship

Fig. 7 illustrates the selected key interaction forces between subsystems. It was observed the longitudinal force acting on the vehicle by the riser tended to decrease from an initial approximately $10 \mathrm{kN}$ 
to nearly 0 and stay at a small value. The longitudinal force acting on the pipeline by the surface ship tended to increase gradually along with sinusoidal variation from the initial approximately $20 \mathrm{kN}$ to $70 \mathrm{kN}$, and will remain stable with the continuous integrated motion of the total system.

The inhomogeneous distribution of the seafloor sediment is a common condition, which will directly cause the vehicle to deviate from its predetermined path. Fig. 8 shows the motion trajectories of subsystems under this condition. The motion trajectories of other subsystems will also deviate, accompanied by the vehicle.

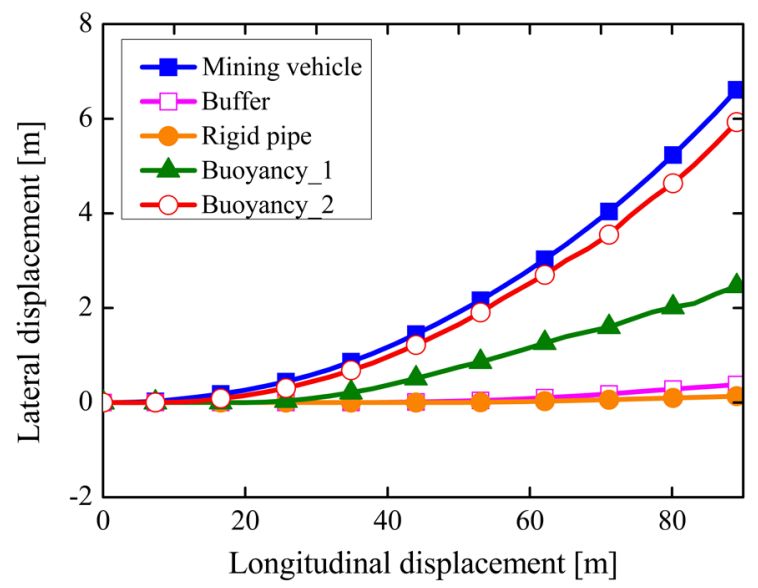

Fig. 8. Trajectories simulations of subsystems for inhomogeneous distribution of the seafloor sediment

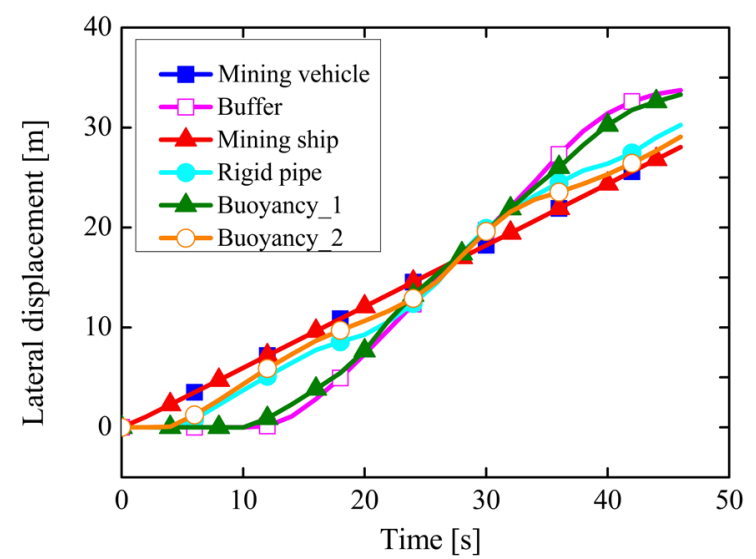

Fig. 9. Displacements simulations of subsystems for the lateral motion

Fig. 9 presents the simulation trajectories of subsystems for lateral motion of the total system. It was observed that the synchronized motion of the total system could be maintained well on the premise that the lateral displacement of the vehicle was within approximately $20 \mathrm{~m}$. Therefore, in the actual operation, the lateral displacement of the total system should be well planned and controlled.

\section{CONCLUSIONS}

A 3D multi-body dynamic simulation model of a seafloor-tracked mining vehicle has been developed with the integration of a user-defined subroutine for the seafloor sediment terramechanics model. This research provides an effective dynamic modelling method for tracked vehicles on various terrains.

An innovative user-defined subroutine for parametrically and rapidly automatically building the long mining pipeline as a multi-body DEM model has been developed in the C\# language in the ProcessNet platform in the RecurDyn environment. This research provides a high-efficient dynamic modelling method for an extremely long pipeline.

A novel multi-body dynamic simulation model of the deep ocean mining vehicle-pipeline-ship system was established in the RecurDyn environment by integrating a new multi-body DEM model of the mining pipeline to a 6-DOFs kinematics model of the ship and a multi-body dynamic model of the seafloor mining vehicle. The integrated motion simulations demonstrate that the total mining system can well maintain a synchronized stable motion state during the whole operations.

\section{ACKNOWLEDGEMENTS}

This research is financially supported by the Foundation Research Project of Shenzhen Science and Technology Program (Grant No. JCYJ20130401160614378) and the State Key Laboratory of Ocean Engineering (Shanghai Jiao Tong University) (Grant No. 201515).

\section{REFERENCES}

[1] Chung, J.S. (2009). Deep ocean mining technology III: Developments. Proceedings of the 8th ISOPE Ocean Mining Symposium, p. 1-7.

[2] Dai, Y., Liu, S.J. (2013). Theoretical design and dynamic simulation of new mining paths of tracked miner on deep seafloor. Journal of Central South University, vol. 20, no. 4, p. 918-923, DOI:10.1007/s11771-013-1566-z.

[3] Dai, Y., Zhu, X., Chen, L.S., Liu, H., Zhang, T., Liu, S.J. (2015). A new multi-body dynamic model for seafloor miner and its trafficability evaluation. International Journal of Simulation Modelling, vol. 14, no. 4, p. 732-743, Dol:10.2507/ IJSIMM14(4)C019.

[4] Rubinstein, D., Hitron, R. (2004). A detailed multi-body model for dynamic simulation of off-road tracked vehicles. Journal of 
Terramechanics, vol. 41, no. 2-3, p. 163-173, D0l:10.1016/j. jterra.2004.02.004.

[5] Rubinstein, D., Coppock, J.L. (2007). A detailed single-link track model for multi-body dynamic simulation of crawlers. Journal of Terramechanics, vol. 44, no. 5, p. 355-364, DOl:10.1016/j.jterra.2007.10.004.

[6] Gao, Y., Wong, J.Y. (1994). The development and validation of a computer aided method for design evaluation of tracked vehicles with rigid links. Proceedings of the Institution of Mechanical Engineers, Part D: Journal of Automobile Engineering, vol. 208, no. 3, p. 207-215, D0l:10.1243/PIME_ PROC_1994_208_183_02.

[7] Wong, J.Y., Huang, W. (2005). Evaluation of the effects of design features on tracked vehicle mobility using an advanced computer simulation model. International Journal of Heavy Vehicle Systems, vol. 12, no. 4, p. 344-365, D0l:10.1504/ IJHVS.2005.008304.

[8] Wong, J.Y., Senatore, C., Jayakumar, P., lagnemma, K. (2015). Predicting mobility performance of a small, lightweight track system using the computer-aided method NTVPM. Journal of Terramechanics, vol. 61, p. 23-32, D0l:10.1016/j. jterra.2015.07.002.
[9] Hong, S., Kim, H.W., Choi, J. (2003). A new method using Euler parameters for $3 D$ nonlinear analysis of marine risers/pipelines. Proceedings of 5th ISOPE Ocean Mining Symposium, p. 83-90.

[10] Rustad, A.M., Larsen, C.M., Sørensen, A.J. (2008). FEM modelling and automatic control for collision prevention of top tensioned risers. Marine Structures, vol. 21, no. 1, p. 80-112, D0l:10.1016/j.marstruc.2007.04.003.

[11] Brink, A.W., Chung, J.S. (1981). Automatic position control of a 300,000-ton ship ocean mining system. Transactions of ASME Journal of Energy Resources Technology, vol. 104, no. 4, p. 285-293, D0I:10.1115/1.3230417.

[12] Hong, S., Kim, H.W. (2005). Coupled dynamic analysis of underwater tracked vehicle and long flexible pipe. Proceedings of the $6^{\text {th }}$ Ocean Mining Symposium, p. 132-140.

[13] Bekker, M.G. (1969). Introduction to Terrain-Vehicle Systems. University of Michigan Press, Michigan.

[14] Janosi, Z., Hanamto, B. (1961). The analytical determination of drawbar pull as a function of slip for tracked vehicles in deformable soils. International Conference of the International Society for Terrain-Vehicle Systems, p. 707-736.

[15] Wong, J.Y. (2010). Terramechanics and Off-Road Vehicle Engineering, Elsevier Science Publishers, B.V. Amsterdam 